\title{
Nonintercepting electron beam size monitor using optical diffraction radiation interference
}

\author{
A. Cianchi, ${ }^{1, *}$ M. Castellano, ${ }^{2}$ L. Catani, ${ }^{3}$ E. Chiadroni, ${ }^{2}$ K. Honkavaara, ${ }^{4}$ and G. Kube ${ }^{4}$ \\ ${ }^{1}$ INFN-Roma Tor Vergata \& University of Rome "Tor Vergata", Via della Ricerca Scientifica, 1-00133 Rome, Italy \\ ${ }^{2}$ INFN-LNF, Via E. Fermi, 40-00044 Frascati, Rome, Italy \\ ${ }^{3}$ INFN-Roma Tor Vergata, Via della Ricerca Scientifica, 1-00133 Rome, Italy \\ ${ }^{4}$ DESY, Notkestrasse 85, 22607 Hamburg, Germany \\ (Received 1 April 2010; published 28 October 2011)
}

\begin{abstract}
In recent years, the use of diffraction radiation (DR), emitted when a charged particle beam passes through a rectangular slit, has been proposed and successfully tested as a nonintercepting diagnostic of high brightness beams. However, some problems related to the control of the particle trajectory through the slit still remain. If an additional slit is placed in front of the first one, at a distance shorter than the radiation formation length, interference between the forward diffraction radiation from the upstream slit and the backward diffraction radiation from the downstream slit can be observed. In this paper we report the first experimental observation of this effect, which we call here optical diffraction radiation interference (ODRI). If the two slits have different dimensions and are not aligned on the same axis, the properties of the ODRI pattern can be effectively used for nonintercepting beam diagnostics, especially for the unambiguously determination of the beam size. Indeed, the advantage of ODRI compared with a single aperture DR screen is due to the reduction of synchrotron radiation background, the increase of sensitivity for transverse beam dimensions, and the possibility to separate effects caused by the beam size and by beam offset within the slit.
\end{abstract}

DOI: 10.1103/PhysRevSTAB.14.102803

PACS numbers: 41.60. $-\mathrm{m}, 07.77 . \mathrm{Ka}, 29.27 .-\mathrm{a}, 41.75 . \mathrm{Ht}$

\section{INTRODUCTION}

Fourth generation free-electron laser (FEL) based light sources and future linac colliders demand high brightness electron beams. Measurement of the beam size along the accelerator is one of the fundamental diagnostics required for such a facility, allowing the control of the beam matching through the machine as well as measurements of the transverse emittance. Conventional diagnostic methods are based on the interaction of the electron beam with intercepting measurement devices. However, high brightness beams, due to their high power density, deposit into such an apparatus an unsustainable amount of energy leading to damage of the device. Therefore developments of parasitic beam diagnostics methods are essential.

When a charged particle passes through the aperture on a boundary between two media with different refraction indices, diffraction radiation (DR) is emitted both into the forward and the backward direction. The DR is emitted only when the dimension of the transverse electromagnetic field, at given wavelength and beam energy, is larger than the aperture size. Since the beam passes through a hole, DR provides a nonintercepting diagnostics tool, and is

\footnotetext{
*cianchi@roma2.infn.it
}

Published by the American Physical Society under the terms of the Creative Commons Attribution 3.0 License. Further distribution of this work must maintain attribution to the author(s) and the published article's title, journal citation, and DOI. therefore well suited for measuring parameters of high charge density beams in a parasitic way.

The physics of DR is well known in the literature [1]. In 1997 one of the authors suggested a method for a nonintercepting measurement of particle beam sizes using DR from a rectangular aperture [2]. Other authors have proposed a similar technique by using a circular aperture [3]. The choice of a rectangular slit shape has many advantages. The mathematical description is simplified due to the translational invariance with respect to one of the coordinates. The size of the slit in that direction can be considered as infinite with respect to the transverse size of the electromagnetic (EM) field (in the order of $\gamma \lambda$, where $\gamma$ is the relativistic factor and $\lambda$ is the wavelength of observation) associated with the traveling electrons. As a consequence, only one coordinate is relevant: the one corresponding to the direction of the displacement of the beam with respect to the slit center.

Here we focus on the incoherent DR emitted at optical wavelengths [optical diffraction radiation (ODR)] using the far field approximation. The feasibility and advantages of this technique have been first demonstrated by measurements performed at ATF at KEK [4-6]. However, these results have also pointed out some difficulties related to the experimental setup, in particular the low ODR signal-tonoise ratio, mainly affected by the unavoidable synchrotron radiation (SR) background [7] produced by the same beam in the upstream magnetic elements of the transport line. Moreover, an accurate and nontrivial control of the beam trajectory is required due to the ambiguity of 
radiation produced by a pointlike beam passing through an aperture off center, and a centered Gaussian distributed beam with a transverse rms (root mean square) beam size equal to this offset with respect to the slit center according to Eq. (6) in Ref. [2].

To overcome these limitations, we performed a theoretical and experimental study with special emphasis on electron beams having an energy higher than several hundreds of $\mathrm{MeV}$, transverse beam sizes below $100 \mu \mathrm{m}$, and integrated charges around or above $1 \mathrm{nC}$.

In a first step we considered radiation from a single slit screen as described in the experiment presented in Ref. [4]. As already found by the authors in this reference, the SR background is very strong compared to the DR signal. The beam pipe acts as a light guide, and so SR produced by upstream magnetic elements is randomly scattered and contributes to the background. Since SR travels together with the electron beam, it is very difficult to filter it out. In a second step we extended our target to a two-slits system (Fig. 1). The first screen with its surface normal to the beam axis acts as a background shielding mask. The second screen, tilted by 45 degrees and with a slit width $b$ smaller than the first slit aperture, behaves as the main DR radiator. However, the radiation generated also from the first screen interferes with the one originating from the second screen.

The idea to use a two-slit system of equal apertures has been first proposed in Ref. [8]. A setup, with one slit which can be rotated in two different orientations perpendicular to each other, can allow in principle the evaluation of the beam size and the angular divergence. However, it does not provide any shield against SR, resulting not suitable in our case due to the background problem described above. In the case of two parallel oriented slits with equal apertures, both oriented at 45 degrees with respect to the beam axis, the authors of Ref. [8] showed that it is possible to determine the beam divergence using DR interferometry. This kind of setup is an ODR analog of the Wartski interferometer for optical transition radiation (OTR) [9]. However, their results also indicate that this kind of setup can only be
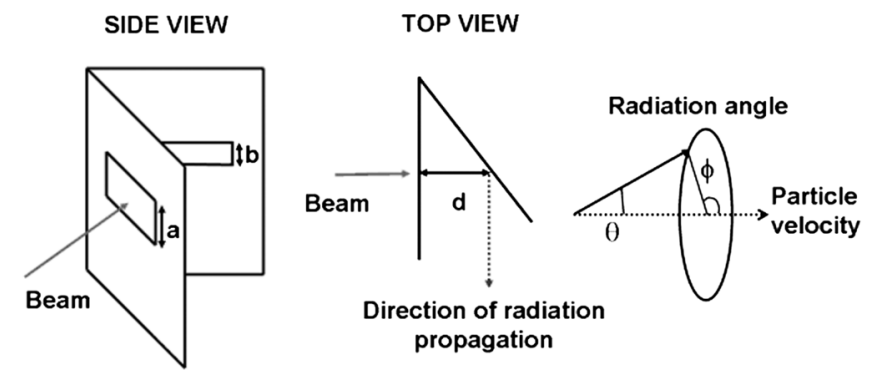

FIG. 1. Sketch of the two-slits setup. Left: side view; center: top view; right: definition of the coordinates system. Dimension $a$ is the aperture size of the first slit, $b$ the size of the second slit, and $d$ the distance between slits center. used when the distance between the screens is at least a significant fraction of the radiation formation length $L$ $\left(L \approx \gamma^{2} \lambda\right)$. Since forward and backward radiation amplitudes sum up with opposite sign, their interference results in an almost complete cancellation of the total intensity when their distance is much shorter than the formation length.

In order to have an appropriate and compact setup, we came up with the idea to use slits with different apertures, placed at a distance much shorter than the formation length. This is a crucial difference between our setup and the others usually used when the interference effects between OTR and ODR are considered: in the standard interferometry setups the distance between the radiators is much longer than the formation length. Therefore the behavior of the interference effect in our case differs from the ones observed with the standard interferometer scheme, for instance that of Ref. [8]. In our scheme, due to the small phase difference between the two screens, the high frequency fringes (with frequency much larger than $1 / \gamma$ ), being very sensitive to the beam angular spread, are not visible. Instead, the main feature of the radiation produced using a single slit is preserved, with modifications in the angular distribution introduced by interference making lower frequency fringes (with frequency in the order of $1 / \gamma$ and produced by the two sides of the slits) more visible, and still sensitive to the beam size.

In our setup the inter-slit distance is only a few centimeters. The choice of the first slit width is a compromise between two requirements. First of all, it has to be small enough to be effective as SR shielding mask. Furthermore, its size cannot be equal or close to the second slit aperture. In such a case the forward diffraction radiation (FDR) and backward diffraction radiation (BDR), emitted from the first and the second interface, interfere negatively resulting in a negligible total intensity. In our case the first and the second slits have widths of 1 and $0.5 \mathrm{~mm}$, respectively. This difference in size is sufficient to generate DR with a clear interference effect. The effect of the first slit is significant not only on the radiation intensity, but also on its angular distribution.

FDR emerging from the first slit travels towards the second screen, which is tilted at 45 degrees with respect to the first one. On the second screen FDR is reflected and superimposed to BDR emitted by this screen. A camera, placed in the focal plane of our optical setup, records the resulting optical diffraction radiation interference (ODRI) angular distribution pattern.

\section{ODRI THEORY}

In order to understand the result of the two-slits interference, DR emitted when a particle of charge $e$ crossing a slit of width $a$ at a distance $y$ from its center [1] is considered. The field component with polarization direction normal to the slit edge is given by 


$$
\begin{gathered}
E_{y}=\frac{i e}{4 \pi^{2} c}\left\{\frac{e^{-[(a / 2)+y]\left(f-i k_{y}\right)}}{f-i k_{y}}-\frac{e^{-[(a / 2)-y]\left(f+i k_{y}\right)}}{f+i k_{y}}\right\} \\
f=\sqrt{k_{x}^{2}+\eta^{2}}, \quad k=\frac{2 \pi}{\lambda}, \quad \eta=\frac{k}{\beta \gamma}, \\
k x=k \sin \theta \cos \phi, \quad k y=k \sin \theta \sin \phi,
\end{gathered}
$$

where $\theta$ and $\phi$ are the polar angles as defined in Fig. 1.

The horizontal DR polarization field component is not considered here because in our experimental setup it is suppressed by a polarizer. Only the vertical polarization is measured.

Considering a single slit setup and $\gamma \gg 1$, the amplitude given by (1) can be used to represent both the FDR with $\theta$ and $\phi$ angles measured with respect to the particle velocity and the BDR with $\theta$ and $\phi$ measured with respect to the reflection direction [10,11]. In the case of a two-slits geometry, as shown in Fig. 1, the FDR and the BDR field amplitudes contribute to the interference pattern with opposite sign. This is the result of a phase difference. In addition to this intrinsic phase difference of $\pi$, there is a phase difference between the two DR amplitudes, which is introduced by the different speed between particle and radiation, and resulting in a slightly delayed emission of $\mathrm{BDR}$ at the second screen with respect to the wave front of FDR emitted at the first slit.

Neglecting the constant coefficient, the vertical component of the total field amplitude produced by the two perfectly centered slits can be written as

$$
\begin{aligned}
E_{y}= & \left\{\frac{e^{-\left[\left(a_{1} / 2\right)+y\right]\left(f-i k_{y}\right)}}{f-i k_{y}}-\frac{e^{-\left[\left(a_{1} / 2\right)-y\right]\left(f+i k_{y}\right)}}{f+i k_{y}}\right\} \\
& -e^{i \Phi_{0}}\left\{\frac{e^{-\left[\left(a_{2} / 2\right)+y\right]\left(f-i k_{y}\right)}}{f-i k_{y}}-\frac{e^{-\left[\left(a_{2} / 2\right)-y\right]\left(f+i k_{y}\right)}}{f+i k_{y}}\right\}
\end{aligned}
$$

with $\Phi_{0}=\frac{2 \pi d}{\beta \lambda}(1-\beta \cos \theta)$ and $d$ the distance between the two slits.

Figure 2 shows the comparison of two angular distributions emitted by a single particle in the case of ODR originating from the second slit only (1), and for the twoslits ODRI setup (2). The distributions are calculated using Eqs. (1) and (2), respectively. It was assumed that the particle passes through the center of both slits with an energy of $\gamma=1840$. The width of the first slit is $a_{1}=$ $1 \mathrm{~mm}$ and of the second $a_{2}=0.5 \mathrm{~mm}$. Their distance is $d=20 \mathrm{~mm}$, and the observation wavelength is $\lambda=$ $800 \mathrm{~nm}$.

In the case of the two-slits setup (Fig. 2), the angular distribution shows both central peaks with lower intensity, and the intensity of the side peaks simultaneously increased compared to the single slit ODR distribution. The increased visibility of the side peaks allows us to use more data points in the fitting procedure, thus easing the determination of the beam size from the angular distri-

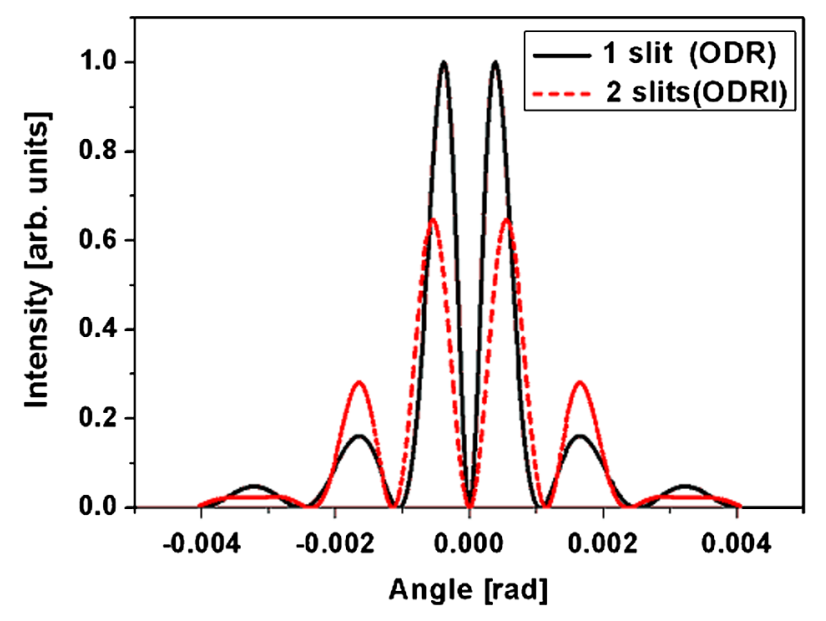

FIG. 2. Comparison between ODR and ODRI angular distributions. Parameters of calculation are indicated in the text.

bution. It is clear from Fig. 1 that in our case the distance $d$ is not constant, but it is a linear function of the horizontal particle position. This fact is unavoidable in our setup. In order to collect the radiation coming from the second screen, the easiest choice is to tilt it at 45 degrees with respect to the beam axis. In such a configuration, the radiation is emitted at 90 degrees with respect to the beam line. The SR background is properly shielded, without losing signal from the second screen, by mounting the first screen perpendicularly to the beam axis.

So the screens are not parallel and thus the distance $d$ between them is not constant. However, since the typical beam size is small compared to $d$, the variation of this parameter is negligible within the transverse dimension of the beam.

In order to give an estimation about the effectiveness of the SR suppression, it is worth mentioning that, in our experimental setup, the last bending magnet is placed more than 40 meters away. As a consequence, only a very small fraction, about $6 \mu \mathrm{rad}$, of the solid angle into which the SR produced by this dipole is emitted, can be captured by the residual part of the second screen which is not shielded by the first one. This value is negligible compared to the angular acceptance of one CCD pixel in our measurement device, which is on the order of $25 \mu \mathrm{rad}$. Even if the SR is produced by quadrupoles upstream of our experiment, since the closest one is placed at about 14 meters from the target, the fraction of the solid angle of SR captured by the screen is smaller than one pixel of our device. Moreover, the very good agreement between the experimental results and the simulation, as well as the comparison between the results retrieved by ODRI analysis and those determined by quadrupole scan, give confidence of the effectiveness of the SR shielding. 
In the case of BDR, a realistic model to be used to validate the experimental data must take into account also a possible small noncoplanarity between the two half planes of the screen. Indeed, as pointed out in [12], even a few tens of nanometers can produce a significant variation in the DR angular distribution. This effect can be included in the model by adding a phase difference between the two terms in Eq. (2), each term being the contribution to the radiation from one half of the screen. For a screen tilted by 45 degrees and $\beta \approx 1$, this phase difference $\Phi_{1}$ is given by $\Phi_{1}=\frac{4 \sqrt{2} \pi \Delta}{\lambda}$ with $\Delta$ being the offset in the longitudinal direction between the two screen half planes. This effect is not relevant for the FDR because both the radiation and the particle travel in the same direction, and their velocity difference is not sufficient to produce any significant phase variation over such a small distance. Indeed the distance between the two screens is too small for diffraction effects from the second slit to influence the free evolution of the forward DR. Therefore, due to the high directionality of the DR at this energy and the small distance between the screens, we assume that the radiation originating from the upper part of the first screen is reflected only by the upper part of the second screen, and the same applies for the lower half of the ODR. It means that any noncoplanarity between the two halves of the second screen results in a time delay, causing a phase difference between the two parts of the FDR as well as with the FDR and the BDR.

In order to have a realistic model for fitting the experimental data, the vertical offset $\delta$ between the slit centers caused by a misalignment has to be introduced as an additional parameter in the ODRI mathematical description. However, rather than being just an error source, the misalignment $\delta$ turns out to be very useful. As mentioned above, the ODR theory [2] states that a Gaussian beam of vertical width $\sigma$ produces exactly the same ODR angular distribution as a pointlike beam traversing the slit with a vertical offset equal to $\sigma$ under the condition $2 f^{2} y^{2} \ll 1$ [Eq. (6) in Ref. [2]]. Even if the former condition is violated, and there is no equivalence between both conditions described above, a systematic error remains in the beam size determination due to the out-of-center position of the charged particle. In the double slit setup, this symmetry is broken due to the misalignment between the two slits, thus the effect of a finite size beam centered within the slit can be distinguished from the one produced by a pointlike misaligned beam. Unlike the single slit ODR, the ODRI measurement does not need a complementary beam position diagnostic to measure the beam vertical offset with respect to the slit center. Indeed, the two parameters (beam size and vertical offset) can be simultaneously determined by a fitting procedure.

Therefore, the general expression for the normal polarization field of the two-slits ODRI is

$$
\begin{aligned}
E_{y}= & \left\{\frac{e^{-\left[\left(a_{1} / 2\right)+y-\delta\right]\left(f-i k_{y}\right)}}{f-i k_{y}}-e^{i \Phi_{1}} \frac{e^{-\left[\left(a_{1} / 2\right)-(y-\delta)\right]\left(f+i k_{y}\right)}}{f+i k_{y}}\right\} \\
& -e^{i \Phi_{0}}\left\{\frac{e^{-\left[\left(a_{2} / 2\right)+y\right]\left(f-i k_{y}\right)}}{f-i k_{y}}-e^{i \Phi_{1}} \frac{e^{-\left[\left(a_{2} / 2\right)-y\right]\left(f+i k_{y}\right)}}{f+i k_{y}}\right\} .
\end{aligned}
$$

This expression is valid for a single particle moving perpendicular to the slit. A real beam consists of many particles having different transverse positions and, due to the beam angular divergence, also different propagation angles. In our analysis we assumed that both the particle position along the vertical axis and the angular spread of the beam are described by Gaussian distributions with rms vertical beam size $\sigma_{y}$ and rms beam divergence $\sigma_{y}^{\prime}$, respectively. A Gaussian distribution is the natural choice for a round beam in a linear accelerator, and in the case of the beam size and shape this is also experimentally validated. We considered also the impact of both horizontal beam size $\left(\sigma_{x}\right)$ and angular divergence $\left(\sigma_{x}^{\prime}\right)$. Since the horizontal size of the slit $(3 \mathrm{~cm})$ is much larger than the radial extension of the EM field associated with a traveling charge $(\gamma \lambda \approx \mathrm{mm})$, the slit can be considered to be infinite in this direction, and therefore the angular distribution does not depend on the horizontal beam size. The choice of a rectangular slit instead of a circular hole simplifies the mathematical treatment of the interference effects and eases the mechanical machining. The disadvantage of this kind of setup is that only one beam dimension can be measured: the one orthogonal to the longer side of the slit, in our case the vertical beam dimension. This drawback, however, could be overcome by using a second setup with slits rotated at 90 degrees with respect to our case.

The horizontal beam angular spread $\left(\sigma_{x}^{\prime}\right)$ gives a contribution to the ODRI angular distribution. In our analysis we consider the angular distribution of vertically polarized ODRI radiation. Both horizontal and vertical beam angular spread give a contribution to it. We have simulated the effect of the horizontal angular spread in our experimental case. Assuming that the angular divergence values in both planes are about $200 \mu \mathrm{rad}$, as it is in our case, the effect of the horizontal spread to the angular distribution is only a scale factor: when the simulated angular distributions without horizontal angular spread and the one with the spread of $200 \mu \mathrm{rad}$ (the vertical spread is $200 \mu \mathrm{rad}$ in the both cases) are normalized according to their maximum values, the distributions are identical. In order to have a difference of the order of a few percents in the central minima (which would still be very difficult to distinguish in the experimental data), the angular spread must be at least $500 \mu \mathrm{rad}$. We would like to point out that the assumption that we can neglect the effect from the horizontal angular spread and include it only in the scaling factor is related to our experimental case and setup, and may not be valid for experiments carried out under different conditions 
concerning the beam energy and the angular divergence, or in the case where a different distance between the slits is used. The effect of the vertical angular spread, as well as the vertical beam size, are included in our model by summing up the distributions given by Eq. (3) for a single particle having different vertical positions inside the slit and different propagation angles.

The effect of the beam angular spread on the DR distribution is described extensively both in Ref. [11] and in Ref. [8]. In Ref. [8] the effect of the angular spread on the DR interference pattern is also discussed, but only in the case of radiation sources separated by more than the formation length. In our case, due to the short distance $(2 \mathrm{~cm})$ between the slits compared to the formation length $(\sim 2 \mathrm{~m})$ at this beam energy, the high frequency fringes do not appear, while the angular divergence (about $200 \mu \mathrm{rad}$ ) of the incoming beam smooths the radiation pattern, as it happens for the angular distribution produced by a single slit.

We studied this effect in detail by performing simulations of the angular distribution produced by a monoenergetic $(\gamma=1840)$ electron beam with several size and divergence values. Our analysis shows that the contribution from the beam size and the beam divergence to the ODRI angular distribution mix with each other, and thus these two effects cannot be clearly identified, at least not for our experimental conditions. However, we also demonstrate that it is possible to solve this problem using noncollinear slits.

Figure 3 shows the central profile of the angular distribution of vertically polarized radiation in the case of a pointlike beam passing through the center of both slits.

Neglecting the intensity dependence which can be taken into account by introducing an overall normalization coefficient, for a system consisting of two collinear slits we can find a set of beam dimension $(\sigma)$ and beam angular divergence $\left(\sigma^{\prime}\right)$ values, where the contributions due to these two parameters produce almost the same ODRI pattern.

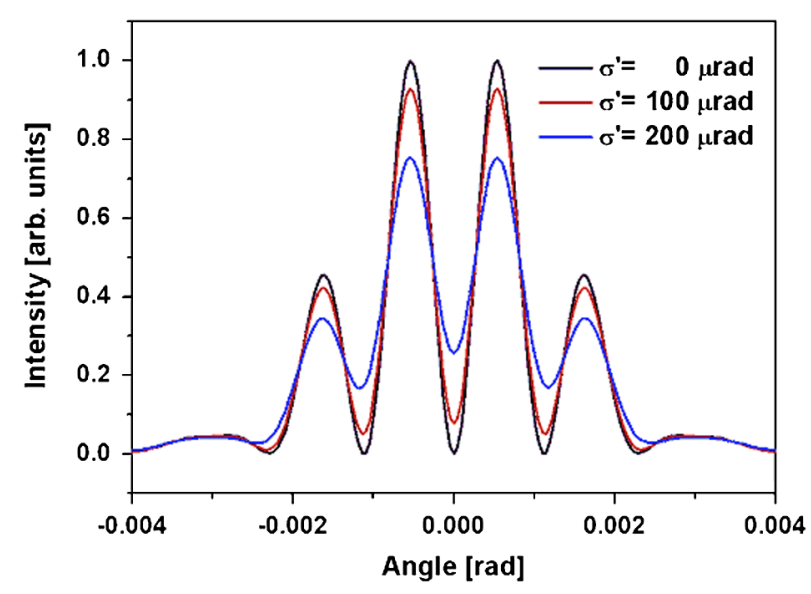

FIG. 3. Comparison between ODRI angular distribution with different values of the angular divergence for a pointlike beam passing in the center of both slits.
This is shown in Fig. 4, which compares angular distributions produced by a pointlike beam having an angular divergence $\left(\sigma=0, \sigma^{\prime} \neq 0\right)$ and a parallel beam having a macroscopic dimension, but no angular divergence $(\sigma \neq 0$, $\sigma^{\prime}=0$ ). The distributions are almost identical, except some differences in the tails, which may even be covered by noise in a real experimental situation. In this case, it is very difficult or even impossible to separate the effects caused by the beam size from that caused by the beam divergence. However, in our experimental setup the slits are not exactly collinear and, as mentioned earlier, this allows a simultaneous determination of the beam size, angular divergence, and vertical offset.

As an example, Fig. 5 shows DR angular distributions produced by three different beams passing through two noncollinear slits (50 $\mu \mathrm{m}$ offset). A parallel beam $\left(\sigma^{\prime}=0\right)$ with a beam size of $\sigma=150 \mu \mathrm{m}$ produces almost identical angular distribution in the central peak region as a pointlike beam $(\sigma=0)$ with an angular divergence of $\sigma^{\prime}=250 \mu \mathrm{rad}$. However, the sideband peaks differ noticeably in these two cases. In the case of a pointlike beam with a divergence of $200 \mu \mathrm{rad}$, the sideband peaks are almost identical to the ones of the parallel beam, but the central peaks differ now substantially. Thus, we can conclude that in the case of our experimental setup consisting of two noncollinear slits, the contributions to the ODRI pattern from the beam size and from the beam angular distribution are significantly different.

Depending on both beam energy and observation wavelength, the slit size is a compromise between two requirements: keep the ODRI signal high enough and preserve the nonintercepting feature of the DR-based diagnostic. Because of the large availability of optical elements and highly sensitive detectors, it is preferable to keep the observation wavelength in the optical range. Furthermore, the lower the beam energy, the larger the geometrical

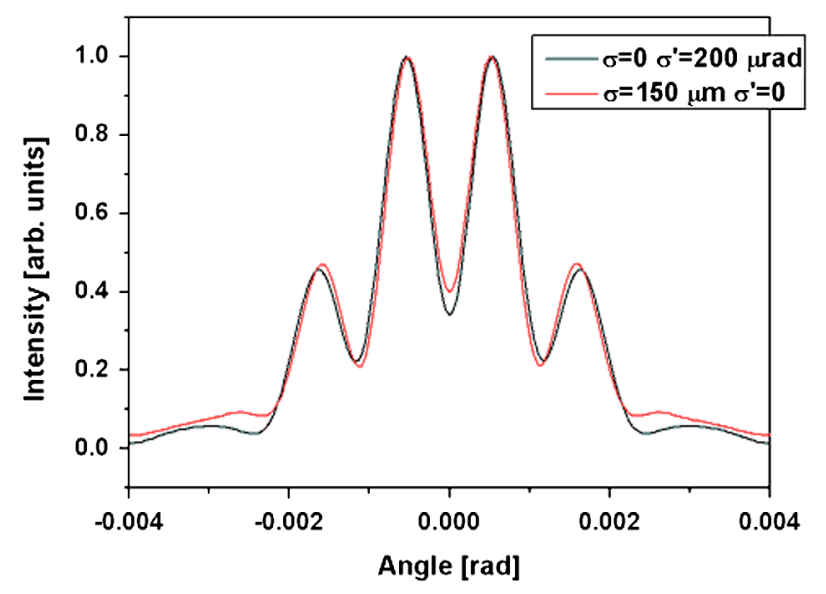

FIG. 4. Angular distribution produced by two different beams passing through a system of two collinear slits: a pointlike beam ( $\sigma=0$, black curve) and a parallel beam, i.e., a beam without angular divergence $\left(\sigma^{\prime}=0\right.$, red curve). 


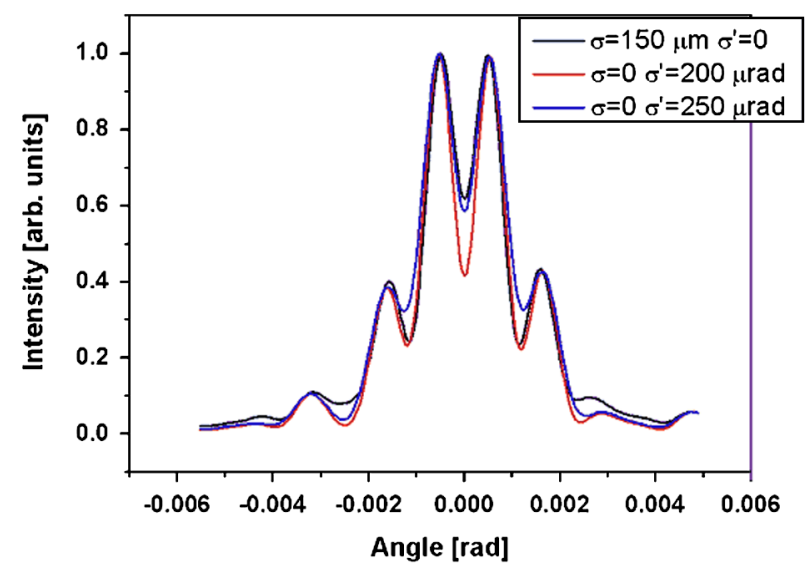

FIG. 5. Angular distribution produced by three different beams passing through a system of two noncollinear slits: a parallel beam with a size of $150 \mu \mathrm{m}$ (black curve), a pointlike beam with angular divergence of $200 \mu \mathrm{rad}$ (red curve), and a pointlike beam with angular divergence of $250 \mu$ rad (blue curve).

emittance is, resulting for a given magnetic lattice, in a larger beam size. This fact draws the line at the minimum allowed slit size, which guarantees a clean transport of the beam through it. For these reasons, the diagnostics technique presented here is well suited for electron beam energies in the $\mathrm{GeV}$ region.

\section{EXPERIMENTAL SETUP}

The measurements presented here have been carried out at FLASH free-electron laser user facility at DESY (Hamburg) [13-15]. FLASH consists of an electron source to produce a high quality electron beam, a superconducting linac with TESLA-type accelerating modules, and an undulator section to produce FEL radiation (see Fig. 6). In addition it has a second electron beam line to by-pass the undulators. Our experimental station is located in this bypass line about $40 \mathrm{~m}$ away from the last bending dipole. This distance is selected to be as long as practically possible in order to minimize the contribution of SR light produced by this dipole. During our measurements, the maximum achievable electron beam energy was about $1 \mathrm{GeV}$, and the maximum number of bunches per bunch train was 30 . The bunch train repetition rate was fixed to

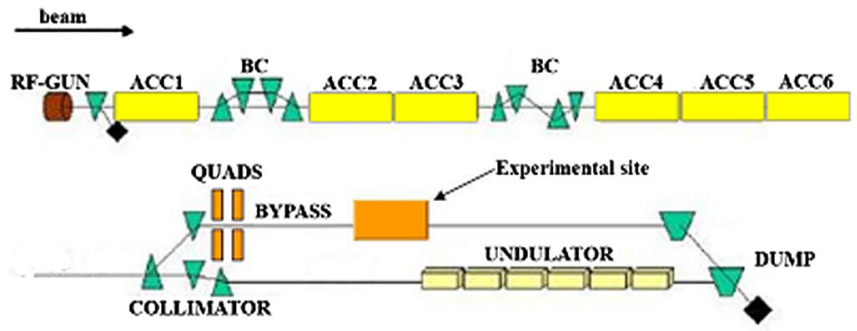

FIG. 6. FLASH layout during our experiment (not to scale). The site of the experiment is indicated.
$5 \mathrm{~Hz}$. In our experiment, we used an electron bunch charge of about $0.8 \mathrm{nC}$. A typical normalized projected transverse rms emittance measured at the injector is around $2 \mathrm{~mm}$ mrad (1 $\mathrm{nC}$ uncompressed bunch). Some emittance growth may occur, when the beam is transported to the by-pass line, where our experiment is located.

Figure 7 shows a sketch of the screen setup. The first screen (mask) is made of stainless steel and acts also as a shield against SR. As mentioned in the previous chapter it is mounted at 45 degrees with respect to the DR target (i.e. normal to the beam direction). The setup includes an additional screen as a spare without any shielding mask. The space $(2 \mathrm{~cm})$ between the two apertures on the second DR screen can be used to determine the transverse beam size by an independent method via imaging the beam using standard OTR.

The DR screen is constructed by a lithographic technique starting from a silicon nitride wafer and opening two slits, one of $0.5 \mathrm{~mm}$ and the other of $1 \mathrm{~mm}$ aperture, by means of chemical etching. The main advantage of the silicon nitride with respect to $\mathrm{SiO}_{2}$ [16] is a much lower etching rate which preserves the silicon substrate from damages and makes the surface much more uniform. An aluminum layer is deposited by sputtering on the target to enhance the reflectivity.

Radiation from the target is reflected by a mirror and transported through an optical system to the camera. Both, an achromatic doublet to image the beam and a biconvex lens with broadband antireflection coating to obtain the DR angular distribution, can be used. The lenses have different focal lengths, $f=250 \mathrm{~mm}$ and $f=500 \mathrm{~mm}$, in order to focus on the same image plane. Two narrow band interference filters, at 800 and $550 \mathrm{~nm}$ and a Glan-Thomson polarizer may be inserted on the optical axis. The polarizer lengthens the optical path, thus increasing the focal length. However, this change is in the range which can be corrected by slightly changing the longitudinal camera

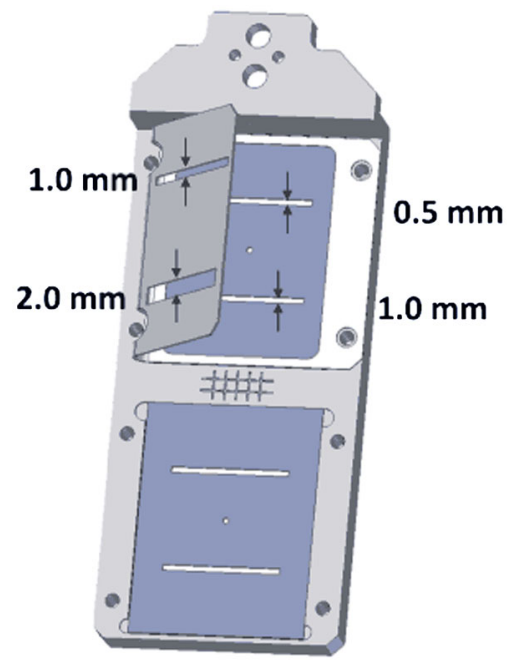

FIG. 7. Sketch of the screen setup. 
position. Because of the very low radiation intensity, a cooled, high sensitivity, 16-bit CCD camera is used (Hamamatsu ORCA II-BT-512G model type C4742-9826LAG2). The camera main features are the very high quantum efficiency in the whole visible spectrum, in particular at $800 \mathrm{~nm}$, the negligible thermal noise, and the long exposure time, up to $2 \mathrm{~h}$.

The optical system is controlled using CAN-bus modules placed in the accelerator tunnel nearby the experiment. In order to allow a remote control, the system is partly integrated in the general control system of FLASH. A PC placed in the tunnel takes care of the camera controls and image acquisition. The PC is connected to a standard Ethernet network, while the local connection between the camera and the PC is realized via a Firewire link.

\section{DATA ANALYSIS}

Data analysis of the ODRI experiment is challenging, since it is not possible to use standard fitting procedures to determine the beam parameters. This is mainly due to the high complexity of the function describing the angular distribution.

The angular distribution of the whole beam is obtained by summing up 5000 distributions produced by a single particle with different vertical positions within the slit and angular divergences, both Gaussian distributed, as already discussed in Sec. II.

Since the resulting expression cannot be solved analytically, a Monte Carlo approach is used instead. Deterministic optimization procedures, like least squares fitting, typically fail when there are several parameters to be adjusted and optimized, in our case including both unknown beam parameters as well as uncertainties on the experimental setup calibration and on the relative position of the slits. In an upgraded experimental setup to be used in the future, some of these parameters, like the offset of the slit centers, can be changed and measured on site, thus simplifying the data analysis.

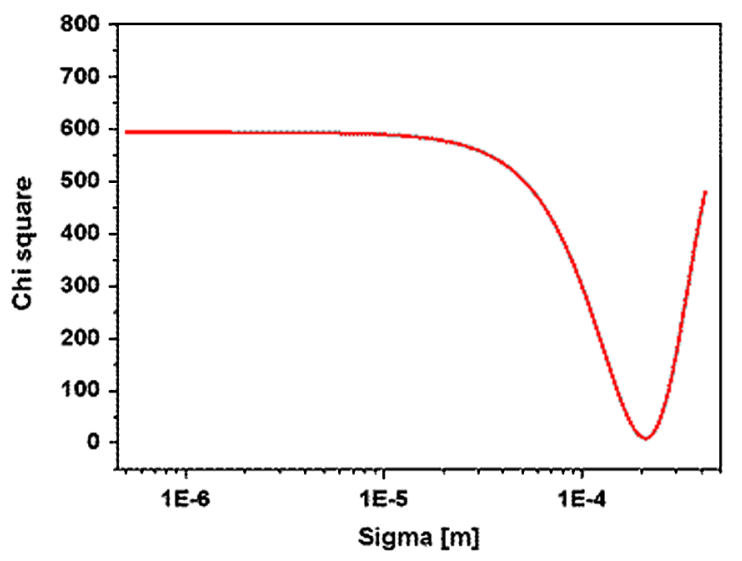

However, the intrinsic stochastic behavior of this distribution may confuse the deterministic fitting procedures, which therefore often fail to find the best set of fitted parameters.

In order to speed up the convergence, a preliminary step to identify an appropriate starting point for the variables and their boundaries can be added to the fitting procedure. We have implemented a search algorithm based on evolutionary strategies [17], in particular on differential evolution, to determine the best starting point for the fit taking into account both the stochastic behavior of the simulated data and the initial wide range of parameters. This search algorithm has been shown to be effective in selecting, for each parameter, the range where the best value for this particular fitting problem is found. Moreover, the differential evolution algorithm is able to find a parameter set which fairly well reproduced the angular distribution. Therefore, a further step of data fitting is not necessary at all.

In a numerical optimization using evolution algorithms, the procedure is based on the minimization of a cost function or the maximization of the score function defined for that particular problem. In our case, the function to be minimized is the point-by-point weight squared difference between the data and fit curves (chi square). The algorithm has been tested with several simulated data sets to verify its ability to identify the best parameter set. The optimization procedure is completed when the used cost function reaches a predefined end value. In our case, this value is reached when the fit curve matches the measured points within their errors (Poisson noise).

From the performed simulations it is evident that the fit is more sensitive to the beam size than to the angular divergence (see Fig. 8).

Uncertainties associated to each fit parameter can in principle be determined by observing the changes of chi square around its minimum value for a given change of the parameter [18]. The resulting uncertainties are typically less than $10 \%$ for beam size and 20\% for angular spread.

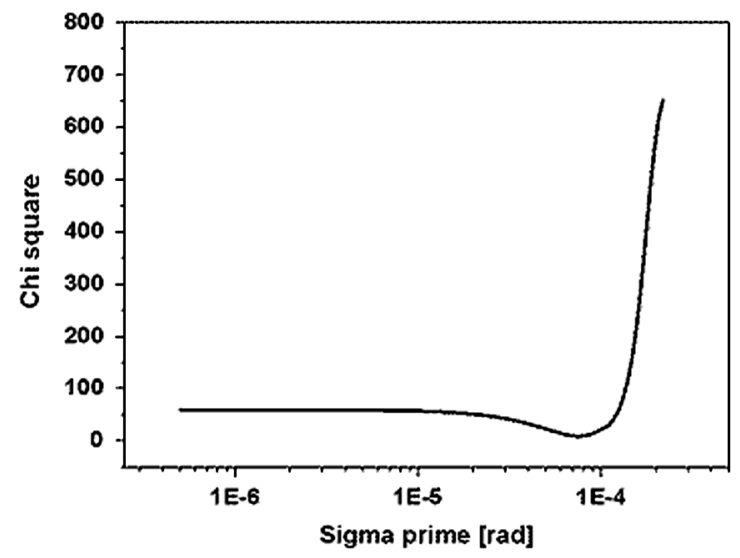

FIG. 8. The best fit parameter set is determined by using a chi-square minimization. Left: chi square vs beam size. Right: chi square vs angular divergence. 


\section{RESULTS}

During the ODRI measurements, FLASH was operated with 13 electron bunches per macropulse and a bunch charge of $0.8 \mathrm{nC}$, at $5 \mathrm{~Hz}$ macropulse repetition rate. The electron beam energy was around $900 \mathrm{MeV}$.

As a first step of the experiment, a complete transverse scan of the beam position over the $0.5 \mathrm{~mm}$ slit aperture has been performed by moving the slit vertically with respect to the beam from one slit edge to the other. The measured angular distributions are shown in Fig. 9 for different beam positions.

As expected, the two slits were not perfectly aligned resulting in a different behavior of the measured angular distribution when the beam moves towards one or the other edge of the second slit. From the data, a top-bottom asymmetry in the angular distribution is clearly visible. This asymmetry can be fully explained by a noncoplanarity of about $70 \mathrm{~nm}$ between the two half planes of the $0.5 \mathrm{~mm}$ slit.

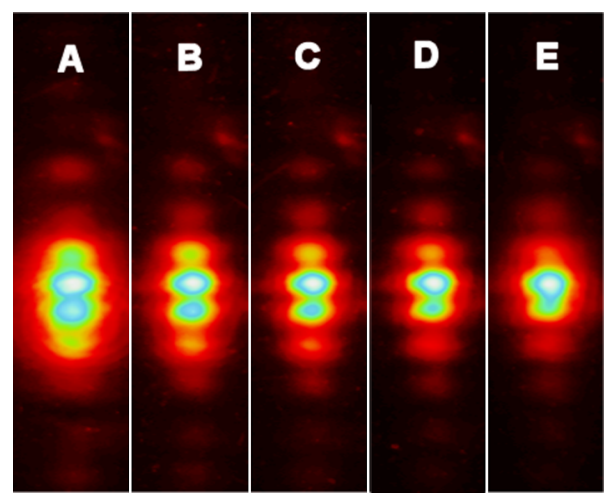

FIG. 9. Images of ODRI angular distribution for different positions of the beam with respect to the center of the $0.5 \mathrm{~mm}$ slit. A: $-125 \mu \mathrm{m} ; \mathrm{B}:-50 \mu \mathrm{m}$; C: center; D: $+50 \mu \mathrm{m}$; E: $+125 \mu \mathrm{m}$.

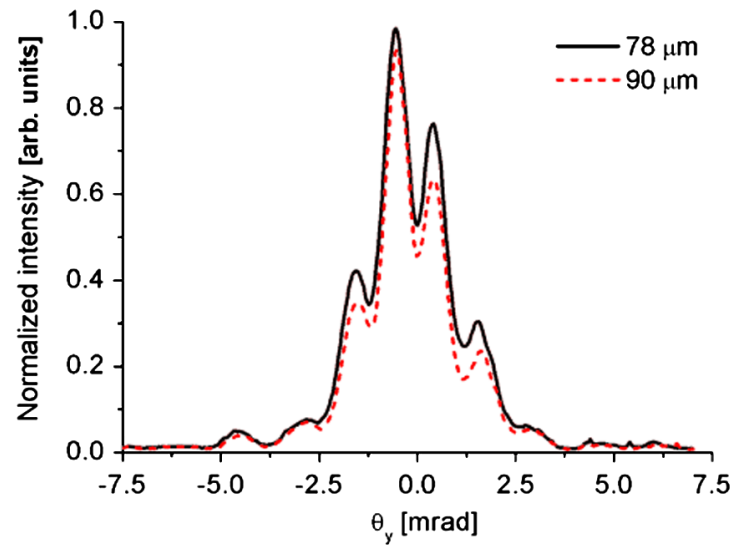

FIG. 10. Measured ODRI angular distributions for two different vertical beam sizes. The beam sizes obtained from the fit are $82 \mu \mathrm{m}$ and $94 \mu \mathrm{m}$. Corresponding beam sizes measured by an OTR monitor are $78 \mu \mathrm{m}$ and $90 \mu \mathrm{m}$ rms, respectively. The associated angular divergences from the ODRI fit are 220 and $200 \mu \mathrm{rad}$, respectively.

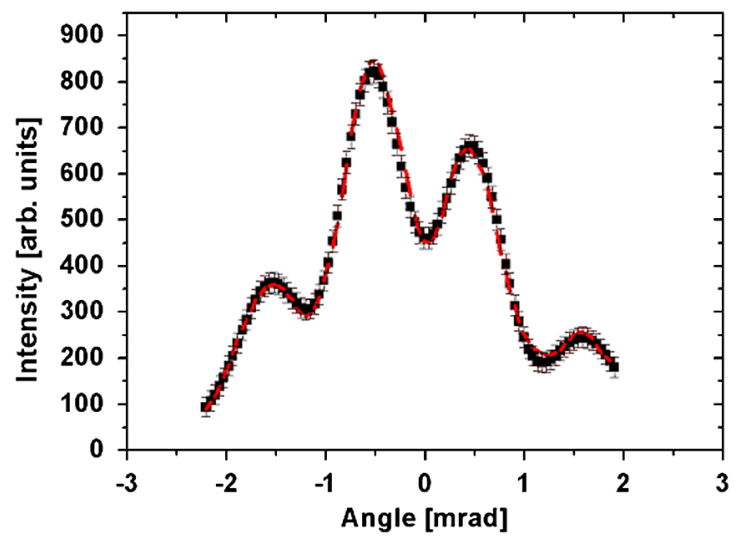

FIG. 11. ODRI angular distribution of a beam going through the center of the second slit. Both experimental data (black squares) and a fit curve, based on theoretical model (red dashed line), are shown.

A mechanical stress introduced by the mask mounting is presumably the reason of the noncoplanarity.

In a second step, the influence of the beam size on the angular distribution was investigated.

Figure 10 shows distributions measured for two different vertical beam sizes. The beam sizes have been determined by the fitting algorithm described above and compared with that obtained by a direct measurement using optical transition radiation.

The contribution of the dark current has been subtracted from the OTR images, and the vertical projection has been fitted by a Gaussian distribution. The beam sizes obtained by the two methods are in good agreement: OTR measurements result in rms beam sizes of $78 \pm 4 \mu \mathrm{m}$ and $90 \pm$ $4 \mu \mathrm{m}$, while the fit of the ODRI distributions provides 82 and $94 \mu \mathrm{m}$, respectively. Despite the small difference in beam size (about 13\%), the two ODRI angular distributions show a detectable difference as can be seen in Fig. 10. The retrieved values for the angular spread are $220 \mu \mathrm{rad}$

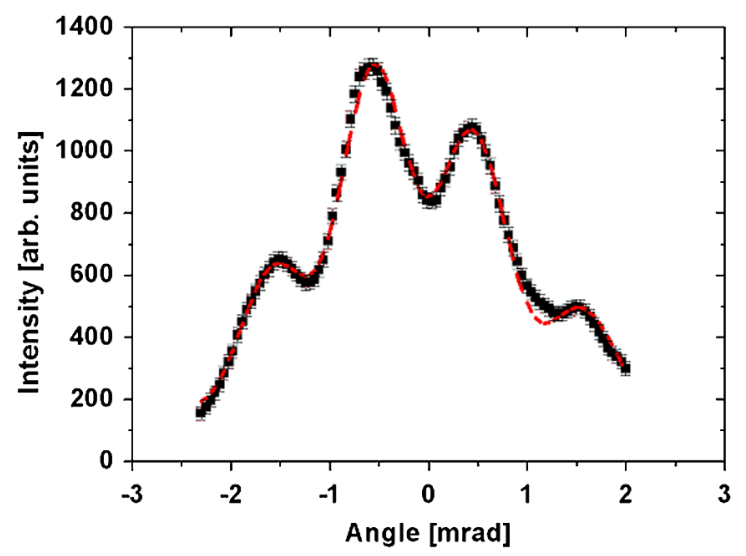

FIG. 12. ODRI angular distribution of a beam going through a slit displaced by $50 \mu \mathrm{m}$ with respect to the former case. Both experimental data (black squares) and a fit, based on the theoretical model (red dashed line), are shown. 
TABLE I. Parameters obtained by fitting the theoretical model to the measured ODRI angular distribution for the two cases shown in Figs. 11 and 12.

\begin{tabular}{|c|c|c|}
\hline & \multicolumn{2}{|c|}{ Figure 11 Figure 12} \\
\hline rms beam size $(\mu \mathrm{m})$ & 82 & 78 \\
\hline rms angular spread ( $\mu \mathrm{rad})$ & 260 & 310 \\
\hline $\begin{array}{l}\text { Displacement of the beam } \\
\text { from the center of second slit }(\mu \mathrm{m})\end{array}$ & -6 & -55 \\
\hline Misalignment between the two slits $(\mu \mathrm{m})$ & 110 & 113 \\
\hline
\end{tabular}

(82 $\mu \mathrm{m}$ beam size) and $200 \mu \mathrm{rad}$ (94 $\mu \mathrm{m}$ beam size). Unfortunately, it was not possible to cross-check the angular spread values with those from a direct measurement of the OTR angular distribution. Indeed, the OTR angular distribution was strongly affected by background radiation, especially in the region of the central minimum of the distribution, which is very important in determining the beam divergence.

In Fig. 11 the experimental data are compared to the theoretical ODRI model by fitting the data in the case when the beam passes through the center of the second slit. It is evident that it is possible to find a parameter set which reproduces extremely well the measured curve. In order to validate the results, a second measurement has been carried out. The slit was moved by $50 \mu \mathrm{m}$ with respect to the beam axis. The agreement between the measured angular distribution and the distribution provided by theory is good also in this case (see Fig. 12). The values, i.e., the beam size, the angular divergence, the displacement from the center of the second slit, and the misalignment between the two slits, obtained from the fit for both cases are compared in Table I. The beam sizes and the misalignment values agree well in the two cases, as well as the displacement of $50 \mu \mathrm{m}$ from the center of the second slit. A divergence of about $320 \mu \mathrm{rad}$ can be estimated from a quadrupole-scan emittance measurement carried out under the same experimental conditions. This value is in very good agreement with the result of the ODRI fit shown in Table I.

\section{CONCLUSIONS}

We have presented the theory and the first nonintercepting beam size measurements using the method, which we call optical diffraction radiation interference (ODRI). Experimental data are consistent with the theoretical model predictions. The beam size values obtained by the analysis of the ODRI angular distribution have been validated by a direct measurement of beam spot size by means of OTR imaging. For some cases, also the beam angular divergence could be compared with an estimate obtained by an alternative method (quadrupole scan) showing a good agreement.

The obtained results demonstrate that measurements using the ODRI effect can provide a basis for nonintercepting high brightness electron beam diagnostics. The main advantages of ODRI, compared to the method using only one DR screen, are the reduction of synchrotron radiation background, increased sensitivity on angular distribution and on beam size, and the possibility to clearly identify and separate the effects caused by the beam size, divergence, and the beam offset within the slit.

\section{ACKNOWLEDGMENTS}

We thank DESY for the opportunity to perform our experiment at the FLASH facility. Special thanks are given to N. Golubeva and V. Balandin for providing the beam optics, and the FLASH operators for their help to setup the machine. We thank also R. Sorchetti and L. Cacciotti from INFN-LNF for their important contribution to the mechanical construction of the experiment setup.

[1] M.L. Ter-Mikaelian, High Energy Electromagnetic Process in Condensed Media (Wiley-Interscience, New York, 1972).

[2] M. Castellano, Nucl. Instrum. Methods Phys. Res., Sect. A 394, 275 (1997).

[3] D.W. Rule, R. B. Fiorito, and W.D. Kimura, in Proceedings of the Seventh Workshop, Argonne, IL, edited by Alex Lumpkin and Catherine E. Eyberger, AIP Conf. Proc. No. 390 (AIP, New York, 1996).

[4] P. Karataev, S. Araki, R. Hamatsu, H. Hayano, T. Muto, G. Naumenko, A. Potylitsyn, N. Terunuma, and J. Urakawa, Phys. Rev. Lett. 93, 244802 (2004).

[5] P. Karataev, S. Araki, R. Hamatsu, H. Hayano, T. Muto, G. Naumenko, A. Potylitsyn, N. Terunuma, and J. Urakawa, Nucl. Instrum. Methods Phys. Res., Sect. B 227, 158 (2005).

[6] T. Muto, S. Araki, R. Hamatsu, H. Hayano, T. Hirose, P. Karataev, G. Naumenko, A. Potylitsyn, and J. Urakawa, Phys. Rev. Lett. 90, 104801 (2003).

[7] G. A. Naumenko, Nucl. Instrum. Methods Phys. Res., Sect. B 201, 184 (2003).

[8] R. B. Fiorito and D. W. Rule, Nucl. Instrum. Methods Phys. Res., Sect. B 173, 67 (2001).

[9] L. Wartski et al., J. Appl. Phys. 46, 3644 (1975).

[10] A. Potylitsyn, Nucl. Instrum. Methods Phys. Res., Sect. B 145, 169 (1998).

[11] N. Potylitsina-Kube and X. Artru, Nucl. Instrum. Methods Phys. Res., Sect. B 201, 172 (2003).

[12] M. Castellano, E. Chiadroni, and A. Cianchi, Nucl. Instrum. Methods Phys. Res., Sect. A 614, 163 (2010).

[13] W. Ackermann et al., Nat. Photon. 1, 336 (2007).

[14] S. Schreiber et al., in Proceedings of IPAC 2010, Kyoto, Japan (ICR, Kyoto, 2010), p. 2149.

[15] S. Schreiber et al., in Proceedings of FEL 2010, Malmoe, Sweden (2010).

[16] E. Cianci et al., Proc. SPIE 4557, 242 (2001).

[17] K. V. Price, R. M. Storn, and J. A. Lampinen, Differential Evolution (Springer, New York, 1998).

[18] I. Hughes and T. Hase, Measurements and their Uncertainties (Oxford University Press, New York, 2010). 